\title{
Biochemistry and genetics of carbohydrate utilization by industrial yeast strains
}

\author{
Graham G. Stewart and Inge Russell \\ Labatt Brewing Company Limited, London, Ontario, Canada, N6A 4M3
}

\begin{abstract}
Saccharomyces cerevisiae and related yeast species have the ability to take up and ferment a wide range of sugars; for example, sucrose, glucose, fructose, galactose, mannose, maltose and maltotriose. In addition Saccharomyces diastaticus is able to utilize dextrins and starch as a result of the secretion of glucoamylase. A major limiting factor in the fermentation of mixed sugar solutions (eg., brewer's wort) is glucose repression. In wort, only after approximately $50 \%$ of the glucose has been taken up will the uptake of maltose commence. In order to overcome this phenomenon, spontaneous mutants of repressed strains have been isolated which are capable of growth in the presence of the non-metabolizable glucose analogue 2-deoxyglucose (2-DOG). These derepressed mutants were found to be capable of maltose uptake in the presence of significant concentrations of glucose. The production of glucoamylase by strains of Saccharomyces diastaticus is also susceptible to carbon catabolite repression by glucose and other sugars. Subjecting such strains to the 2-DOG selection technique has resulted in the isolation of stable spontaneous mutants derepressed for the formation of glucoamylase.
\end{abstract}

\section{INTRODUCTION}

The production of ethanol by microorganisms as a result of the fermentation of sugars and starch is a process that precedes recorded history. Although by far the largest volume of ethanol produced via fermentation is employed for potable purposes, an increasing volume of fermentation ethanol is being produced for fuel ethanol energy either on its own or more usually admixed with gasoline. The production of fermentation ethanol for fuel energy is currently of interest in the United States, Brazil, Argentina, and a number of other countries (ref. 1).

The manufacture of beer is a biological process whereby agricultural products, such as barley and hops, are converted into beer by control of the biochemical reactions in malting, mashing and fermentation. The first two processes together produce a medium known as wort; to this yeast is added, and fermentation is allowed to proceed. Wort is essentially an aqueous extract of malted barley, the primary raw material in the manufacture of beer. Yeast cells are added to the wort and the cells take up nutrients and utilize them so as to increase the yeast population. In doing this, the cells excrete major end-products such as ethanol and carbon dioxide into the medium, together with a host of minor metabolites, many of which contribute to the overall flavour of the final beer.

Until recently, the brewing industry has traditionally been conservative when dealing with yeast performance optimization. Rather than dwelling exclusively on a single fermentation product yield (eg., ethanol), the brewer has quite rightly placed greater emphasis on the flavour characteristics of the final product. Brewery fermentations are slow, nonagitated and at below ambient temperatures; quicker fermentations at higher temperatures or under agitated conditions tend to produce severe off-flavours (due to a plethora of compounds including fusel oils, esters and sulphur compounds) in the beer. However, in the production of fermentation industrial ethanol, the formation of a potable product is not an issue and, as a consequence, the fermentations are usually conducted at, or above, ambient temperatures and are invariably agitated.

There are a number of microorganisms (yeast and bacteria) that produce significant (greater than $1 \% \mathrm{w} / \mathrm{v}$ ) quantities of ethanol (ref. 2). However, at the present time, in excess of $95 \%$ of the fermentation ethanol produced globally involves the use of Saccharomyces cerevisiae and its related species. No other group of microorganisms has been more intimately associated with the progress and well being of the human race than the yeasts. Their contribution to man's progress has been based, very largely, on the capacity of certain 
yeasts to rapidly and efficiently convert sugars to ethanol and carbon dioxide via the alcoholic fermentation of sugary liquids such as grain extracts, grape juice and milk. addition, there are a number of recently developed industrial uses for yeast including cultures that have been genetically transformed to produce important non-yeast proteins and peptides, such as, the antiviral protein interferon (ref. 3), human serum albumin (ref. 4), and the acid protease chymosin employed in the milk-clotting step in cheese production.

\section{WORT SUGAR UPTAKE}

Brewer's wort contains the sugars, sucrose, fructose, glucose, maltose and maltotriose together with dextrin material. In the normal situation, brewing yeast strains are capable of utilizing sucrose, glucose, fructose, maltose and maltotriose in this approximate sequence, although some degree of overlap does occur, leaving maltotetraose and other dextrins unfermented (Fig. 1). A major limiting factor in wort fermentation rates is the repression of maltose and maltotriose uptake by glucose (ref. 5). Only when approximately $50 \%$ of the wort glucose has been taken up by the yeast will the uptake of maltose commence. In other words, in most strains of Saccharomyces cerevisiae and related species, maltose utilization is subject to control by carbon catabolite repression, such that even in the presence of maltose, the maltose-utilizing system will be inactivated by high concentrations of glucose. In order to illustrate this phenomenon, a brewing strain of Saccharomyces cerevisiae has been studied in which the presence of glucose repressed the uptake of maltose. In an attempt to isolate spontaneous mutants of this strain in which the presence of glucose in the medium did not repress maltose uptake, mutants capable of growth in the non-metabolizable glucose analogue 2-deoxyglucose (2-DOG) were isolated (ref. 6). A number of stable 2-DOG mutants were found to be capable of utilizing maltose in the presence of significant concentrations of glucose. For example, 2-DOG resistant mutants of an ale brewing strain of Saccharomyces cerevisiae in $12^{\circ} \mathrm{P}$ wort exhibited increased fermentation rates when compared to the parental strain with complete fermentation being achieved in 45 hours compared to 65 hours in the parental strain (Fig. 2).

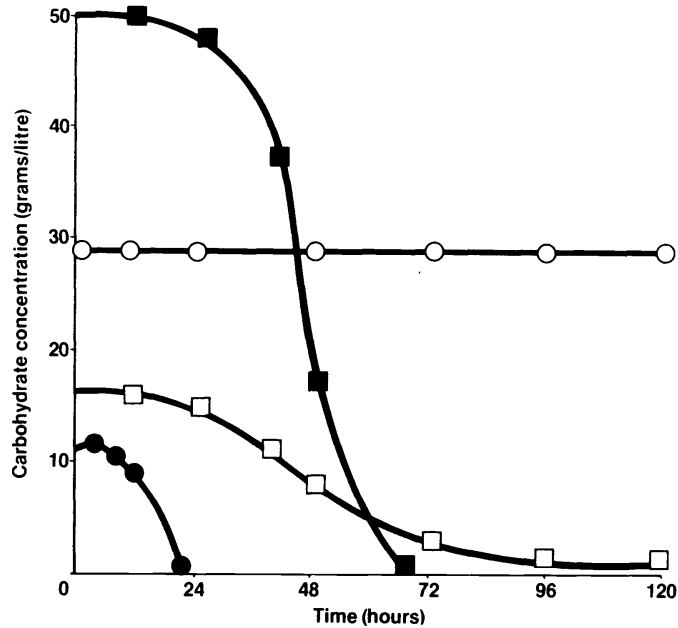

Fig. 1 Uptake of the major wort sugars during fermentation by yeast: maltose ( $)$ ), maltotriose ( $\square-\square$ ), glucose $(\sim)$, dextrin $(\mathrm{O}-\mathrm{O})$.

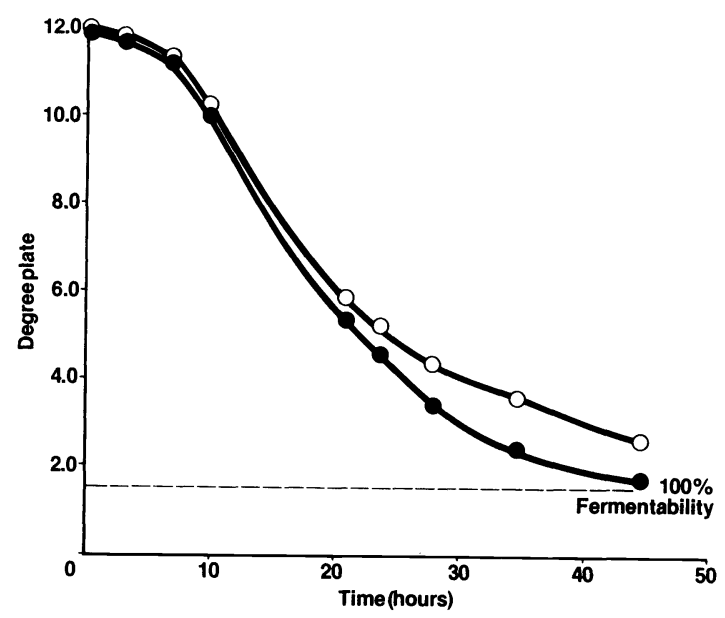

Fig. 2 Fermentation of a $12^{\circ} \mathrm{P}$ wort by parent brewing strain 3001 $(.0-0)$ and its derepressed maltose mutant $(\multimap)$ ). 


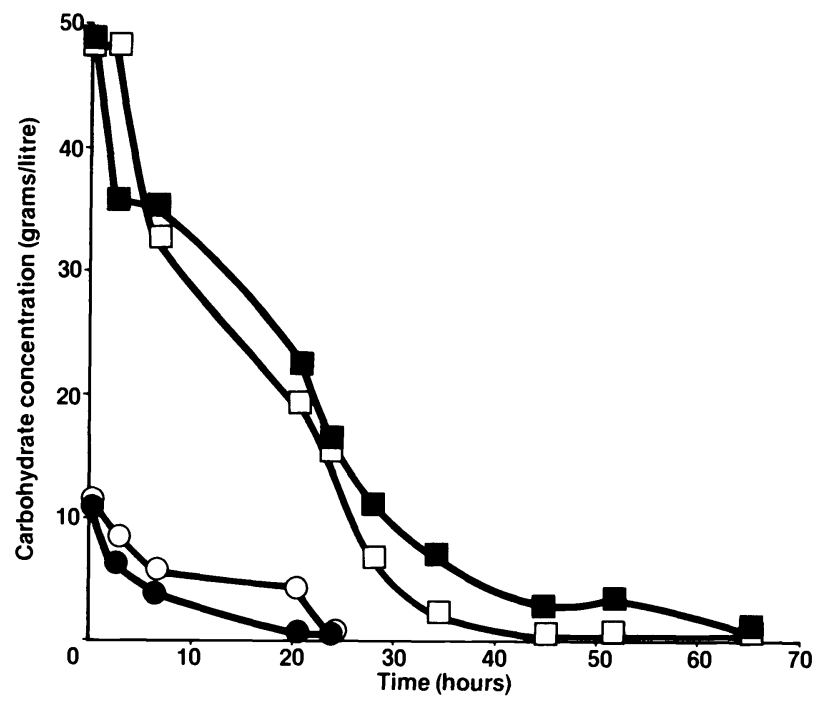

Fig. 3 Carbohydrate uptake in a $12^{\circ} \mathrm{P}$ wort. Parent brewing strain: glucose $(\multimap$ ), maltose ( - ). Derepressed mutant: glucose $(0 \rightarrow)$, maltose $(\square-\square)$.

A trained taste panel, using the triangle test method, determined that the beer produced with 2-DOG mutants was not significantly different from that produced employing the parental yeast strain; all beers were produced under similar pilot plant brewing conditions.

The mechanisms by which 2-DOG resistant mutants are derepressed is far from understood but such mutants have been reported to possess diminished levels of hexokinase (refs. 7,8 ). Possibly hexokinase is associated with a general regulatory system in yeast, involving overall repression. Bailey and Woodword (ref. 9) have described a mutant allele (designated $\mathrm{grr}-1$ ) in Saccharomyces cerevisiae that is characterized not only by 2-DOG resistance, but insensitivity to glucose repression for invertase, $\alpha$-glucosidase (maltase) and galactokinase, as well as the mitochondrial enzyme cytochrome $\underline{c}$ oxidase. The levels of hexokinase activity towards both fructose and glucose were approximately three-fold higher in mutant clones. At this time the molecular mechanism by which catabolite repression is effected in yeast is unknown although many theories have been discussed. Nevertheless, derepressed mutants of industrial yeast strains will be of significant economic importance particularly with respect to increased control of fermentation rate.

\section{LOW CARBOHYDRATE (LOW CALORIE) 'LITE' BEER}

Low carbohydrate (usually low calorie) beer in North America represents a significant share of the beer volume; it constitutes $13 \%$ of the market or 2.2 billion liters per year in the U.S.A. and $8 \%$ or 170 million liters per year in Canada.

Several procedures for producing low carbohydrate beers are being employed or contemplated, most of these center on one of the following techniques: 1) dilution of regular strength beer with water; 2 ) addition of fungal $\alpha$-amylase or glucoamylase with bacterial pullulanase to the wort during fermentation; 3 ) use of glucose, fructose and sucrose as an adjunct; 4) use of brewing yeast strains with amylolytic activity; and, 5) use of a malt enzyme preparation during mashing or fermentation. A further possibility would be to employ a yeast strain that possesses diastatic activity.

As previously discussed, typical brewing yeast strains are capable of utilizing sucrose, glucose, fructose, maltose and maltotriose, leaving the maltotetraose and the larger dextrins unfermented. However, yeasts of the species Saccharomyces diastaticus (ref. 10) have been classified as a distinct species from that of Saccharomyces cerevisiae due to the fact that the former produces the extracellular enzyme glucoamylase, also called amyloglucosidase $(\alpha-1,4$ glucan glucohydrolase, E.C.3.2.1.3.). This enzyme possesses the ability to cleave $\alpha-1,4$ and in some cases $\alpha-1,6$ linkages, releasing glucose from the non-reducing end of starch chains. On the other hand, $\alpha$-amylase $(\alpha-1,4$ glucan 4 glucanohydrolase, E.C.3.2.1.1) is an enzyme which hydrolyzes random $\alpha-1,4$ glucosidic linkages by-passing $\alpha-1,6$ bonds. Three genes have been identified that are associated with glucoamylase production of Saccharomyces diastaticus, DEX1, DEX2 and STA3 (ref. 10). Recently, studies by Erratt and Nasim (ref. 11) have ascertained allelism between DEXI and STA2 and DEX2 and STA1 and it was proposed that in future the two nomenclatures be consolidated. 


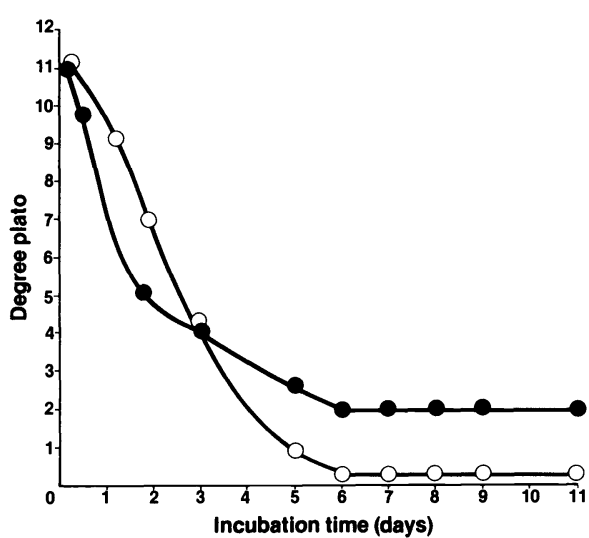

Fig. 4 static fermentation of an $11.3^{\circ} \mathrm{P}$ wort ( 40 litre scale) by a Saccharomyces cerevisiae brewing strain ( - and a Saccharomyces diastaticus diploid (DEX1/DEX1, DEX2/DEX2, STA3/STA3) ( 0 ).

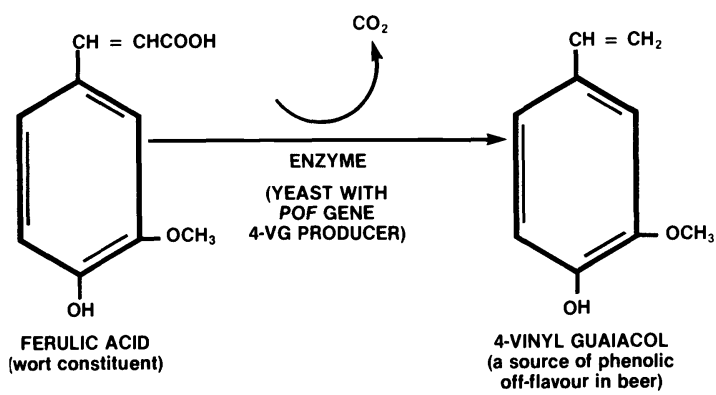

Fig. 5 Enzymatic decarboxylation of ferulic acid to 4-vinyl guaiacol.

Using classical hybridization techniques, a diploid strain containing the DEX and STA genes in the homozygous condition, has been constructed and its fermentation rate studied in brewer's wort under static fermentation conditions (Fig. 4). The initial fermentation rate of this strain was slower than a production ale brewing strain, however, the DEX-containing strain fermented the wort to a greater extent than the brewing strain due to the partial hydrolysis of the dextrins by the action of glucoamylase. Thus, Saccharomyces diastaticus strains possess the capacity to produce beer which has been fermented to a high degree, this property is desirable in the production of low carbohydrate beer; however, it should be noted that beer produced by these strains has a characteristic phenolic-off-flavour. Phenolic-off-flavours in beer are due, on many occasions, to the presence of 4-vinyl guaiacol (4-VG) which arises by the enzymatic decarboxylation of ferulic acid (Fig. 5), a wort constituent (ref. 12).

It has been found by Tubb et al. (ref. 13) that a single dominant nuclear gene designated POF (phenolic-off-flavour) codes for the ferulic acid decarboxylation enzyme. Therefore, strains possessing the POF gene can produce the enzyme capable of decarboxylating ferulic acid. Whereas, brewing Saccharomyces strains normally cannot decarboxylate ferulic acid, all the Saccharomyces diastaticus strains initially studied produced 4-VG in the presence of ferulic acid. Assuming that the POF and DEX genes are independent characteristics, it could be possible to construct a strain containing the DEX but not the POF gene by means of hybridization. Thus, a haploid that was DEX positive and that carried the POF characteristic was mated with a dextrin negative phenolic-off-flavour negative haploid. The resultant diploid fermented dextrin and decarboxylated ferulic acid. When tetrad dissection was carried out a 2:2 segregation for dextrin fermentation and a 2:2 segregation for phenolic-off-flavour was obtained. The DEX and POF genes segregated independently of each other, therefore it was possible to select haploids that were DEX positive and POF negative. Subsequently, a diploid with the genotype DEX2/DEX2, pofo/pofo was constructed and a fermentation of an $11.3^{\circ}$ Plato wort was conducted. Although the initial wort attenuation rate was found to be slower than that of a polyploid ale yeast strain, the yeast was capable of superattenuating the wort, ie., it was able to hydrolyze part of the dextrins into glucose which is readily fermentable, whereas the brewing strain was unable to utilize the dextrins. "Expert" taste panel assessment has deemed the beer produced from this dextrin positive diploid to be rather winey and to have a slightly sulfury character, however, the characteristic phenolic-off-flavour associated with the POF gene (4-VG) could not be detected.

The production of glucoamylase by strains of Saccharomyces diastaticus is subject to carbon catabolite repression by glucose and other sugars (ref. 14) (Fig. 6). Subjecting two such strains to the 2-DOG selection technique, stable spontaneous mutants derepressed for the formation of glucoamylase and mutants derepressed for maltose utilization have been isolated. In $12^{\circ} \mathrm{P}$ wort with a 2-DOG starch mutant of Saccharomyces diastaticus (strain 1393), the level of glucoamylase was increased five-fold when compared to the parental strain (Fig. 7). The overall fermentation rate of the starch mutant was increased when compared to the parental strain (Fig. 8). 


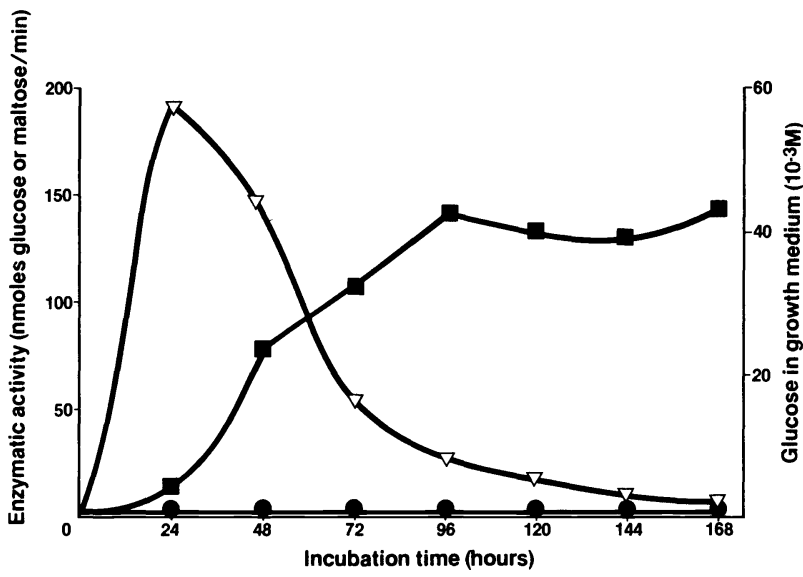

Fig. 6 Amylolytic enzymes produced by Saccharomyces diastaticus strain 1384 during growth in soluble starch: $\alpha-$ amylase activity ( $\bullet$ ), glucoamylase activity ( $\rightarrow$ ), glucose concentration in growth medium $(\nabla \longrightarrow \nabla)$.

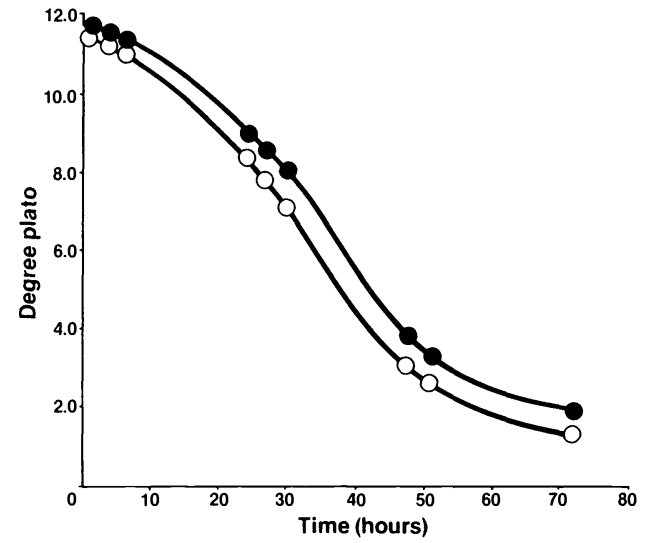

Fig. 8 Fermentation of a $12^{\circ} \mathrm{P}$ wort by a Saccharomyces diastaticus diploid $(\bullet)$ and its starch derepressed mutant $(0-0)$.

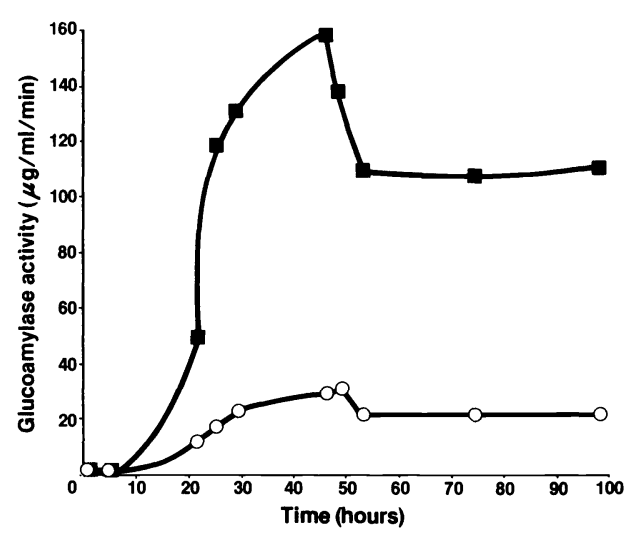

Fig. 7 Glucoamylase production in a $12^{\circ} \mathrm{P}$ wort: parent strain 1393 ( $O-0)$, derepressed starch mutant of strain 1393 ( $\rightarrow$ ).

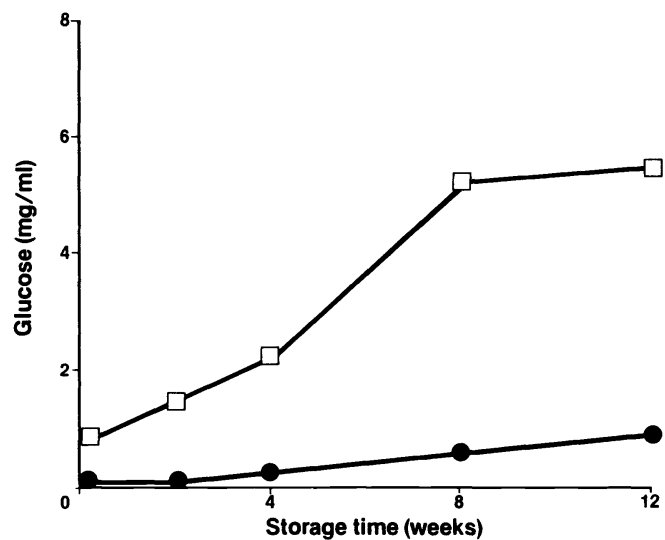

Fig. 9 Production of glucose in pasteurized beer during storage at $21^{\circ} \mathrm{C}$ : Saccharomyces diastaticus strain (DEX) ( $\square-\square)$, Saccharomyces cerevisiae strain ( $\underline{\text { dex }})(\bullet)$ ).

A further problem with the use of strains of Saccharomyces diastaticus (POF or pof) for the production of beer is the fact that the extracellular glucoamylase is heat stable and remains active in the packaged beer after pasteurization. As a consequence, upon storage at $21^{\circ} \mathrm{C}$ an increasing concentration of glucose can be found in the beer (Fig. 9).

This laboratory is committed to achieving the objective of obtaining a strain of Saccharomyces Sp., that possesses the ability to hydrolyze starch entirely, i.e., able to synthesize and secrete $\alpha$-amylase and glucoamylase with debranching ability (ref. 15). Although Saccharomyces diastaticus produces a thermostable glucoamylase, no traces of $\alpha$-amylase or debranching ability could be detected (Table 1 ). 
Table 1. Production of Amylolytic Enzymes by Several Yeast Species

\begin{tabular}{|c|c|c|c|}
\hline \multirow[b]{2}{*}{ Yeast Species } & \multirow[b]{2}{*}{$\alpha$-Amylase } & \multicolumn{2}{|c|}{ Enzymatic Activity } \\
\hline & & Glucoamylase & Debranching \\
\hline $\begin{array}{l}\text { Saccharomyces diastaticus } \\
(1384)\end{array}$ & - & +++ & - \\
\hline $\begin{array}{l}\text { Endomycopsis fibuligera } \\
(240)\end{array}$ & + & ++ & - \\
\hline $\begin{array}{l}\text { Endomycopsis fibuligera } \\
\text { (241) }\end{array}$ & + & ++ & - \\
\hline Pichia burtonii (222) & ++ & + & $+1-$ \\
\hline $\begin{array}{l}\text { Schwanniomyces castellii } \\
(1402)\end{array}$ & ++ & ++ & ++ \\
\hline $\begin{array}{l}\text { Schwanniomyces occidentalis } \\
(1401)\end{array}$ & ++ & ++ & ++ \\
\hline Aspergillus oryzae ( 245 ) & ++ & + & - \\
\hline
\end{tabular}

++ high activity ++ medium activity + low activity - no activity

A strain of Pichia burtonii produced very low levels of debranching activity, however, the yeasts Schwanniomyces castellii and Schwanniomyces occidentalis produced significant amounts of $\alpha$-amylase, glucoamylase and debranching activity. These amylolytic systems have been isolated, purified and characterized and it has been found that schwanniomyces castellii possesses a glucoamylase with debranching activity that is heat labile (ref. 16). Attempts to introduce a number of these amylase systems into saccharomyces spp. are currently ongoing in this laboratory.

As starch is a polysaccharide composed of two polymers, 20-25\% in the form of amylose (linear chains of $\alpha-1,4$ linked glucose residues) and 78-80\% in the form of amylopectin ( $a$ highly branched polymer occurring by $\alpha-1,6$ linkages) debranching activity is essential for complete hydrolysis of the polysaccharide. Two Endomycopsis fibuligera strains studied were found to possess $\alpha$-amylase and glucoamylase activity but no debranching activity (Table 1 ).

\section{DIASTATIC YEASTS FOR DISTILLED ETHANOL PRODUCTION}

As previously discussed, the fermentation of starches to ethanol by yeasts requires pretreatment of the substrate in order to produce fermentable sugars. This pretreatment consists of three steps: gelatinization, liquefaction and saccharification. Gelatinization requires heat and free water and must precede liquefaction. Liquefaction, the dispersion of starch molecules into an aqueous solution, is accomplished by the use of heat and amylolytic enzymes. Heat stable bacterial $\alpha$-amylases or malt enzymes may be employed. During liquefaction, starch molecules are only partially hydrolyzed producing a form of carbohydrate which cannot be assimilated by ethanol-producing yeasts such as Saccharomyces cerevisiae. Therefore, the partially hydrolyzed starch molecules must be converted to lower molecular weight sugars such as glucose and maltose by a 
process known as saccharification. This may be accomplished enzymatically, usually by the addition of fungal glucoamylases to the fermentation vessel at the time of inoculation. The saccharifying glucoamylases represent a significant fraction of the total cost of producing ethanol. Reduction of the amount of added glucoamylase could significantly decrease the cost of the final product.

It has been found possible to decrease glucoamylase addition to starch mash fermentation by employing yeast which actively produce and secrete glucoamylase eg., strains of Saccharomyces diastaticus (refs. 17, 18). A fused genetically manipulated strain of Saccharomyces diastaticus (ref. 19) (strain 1400) was studied as the glucoamylase producing strain and compared to a strain of Saccharomyces cerevisiae (strain 254) which was unable to produce and secrete glucoamylase. The fermentation performance of the two strains was compared in a corn mash with and without added glucoamylase. When the Saccharomyces diastaticus culture was employed, added glucoamylase concentrations could be significantly decreased without reducing ethanol production or sugar uptake (Fig. 10).
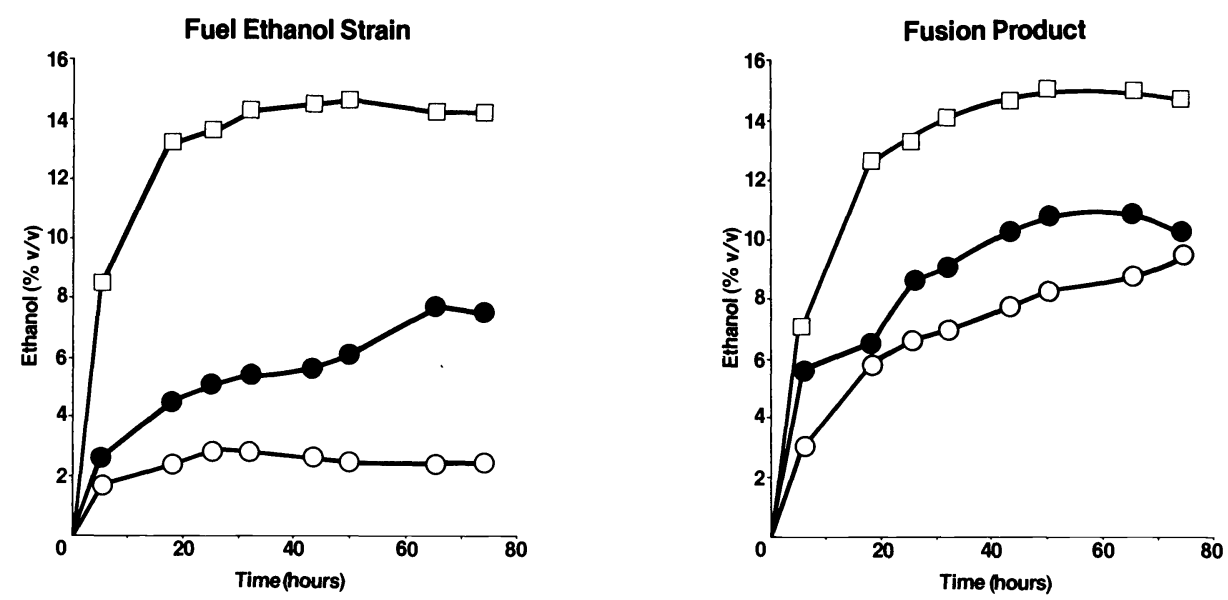

Fig. 10 Ethanol production in a cassava mash with varying amounts of added glucoamylase (AMG). $0.00 \%(0-0), 0.01 \%(\bullet), 0.10 \%$ ( $\square-\square)$.

Reduction of the added glucoamylase concentration from $0.08 \%$ volume, based upon the substrate weight, to $0.03 \%$ resulted in no significant decrease in ethanol yield or sugar uptake; the reduction of added glucoamylase being possible because the fused yeast is able to produce and secrete its own glucoamylase.

Production of glucoamylase by the Saccharomyces diastaticus strain and subsequent reduction of added glucoamylase represents a potential financial saving for the ethanol producer. Since the cost of glucoamylase may exceed $\$ 3.75$ (U.S.) per litre, a 50\% reduction of this enzyme can result in a significant decrease in cost. For example, an ethanol manufacturer that is producing 100 million litres of $95 \%$ ethanol per year uses 190 million $\mathrm{kg}$ of starch (assuming that the fermentation of $20 \%(w / v)$ starch yields $10 \%(\mathrm{v} / \mathrm{v})$ ethanol). For 190 million $\mathrm{kg}$ of starch, 0.19 million litres of glucoamylase are required (based upon an addition of 0.1 litre of glucoamylase per $100 \mathrm{~kg}$ of starch). This results in an annual enzyme cost of approximately $\$ 700,000$ (U.S.) assuming an enzyme cost of $\$ 3.75$ U.S. per litre. Reduction of the added glucoamylase by $50 \%$ represents an annual saving of approximately $\$ 350,000 \mathrm{U}$.S.

\section{CONCLUSIONS}

Although yeast is the oldest of the biotechnology microorganisms, it is still a very important microorganism that is being employed in the modern biotechnology revolution. Current research is being directed into two areas, the first being to improve its capacity and efficiency to produce ethanol and carbon dioxide from a wide variety of substrates and the second is to employ yeast to synthesize and excrete a wide variety of proteins or peptides that posses therapeutic and commercial potential. 


\section{Acknowledgements}

Part of the research from this laboratory that is discussed was funded by a PILP contract from the National Research Council of Canada which is gratefully acknowledged.

\section{REFERENCES}

1. G.G. Stewart, The World Biotechnology Report USA 2, 98-119 (1984).

2. G.G. Stewart, CRC Crit. Revs. in Biotechnol. 1, 161-176 (1984).

3. R.A. Hitzman, D.W. Leung, L.J. Perry, J.W. Kohr, H.L. Levine and D.W. Goeddel, Science 219, 620-626 (1983).

4. S.W. Molzahn, European Patent Application No. 0147198 A2 (1984).

5. G.G. Stewart, I. Russell and A.M. Sills, MBAA Tech. Quart. 20, 1-8 (1983).

6. G.G. Stewart, R. Jones and I. Russell, Europ. Brew. Conv. Proc. 20th Congress, Helsinki, Finland 243-250 (1985).

7. K.D. Entian, F.K. Zimmerman and I. Scheel, Mol. Gen. Genet. 156, 99-105 (1977).

8. E.A. Pfisterer, I.F. Garrison and R.A. McKee, MBAA Tech. Quart. 15, 59-63 (1978).

9. R.B. Bailey and A. Woodward, Mol. Gen. Genet. 193, 507-512 (1984).

10. J.A. Erratt and G.G. Stewart, in Current Developments in Yeast Research (G.G. Stewart and I. Russell, eds.) p.177-183, Pergamon Press, Toronto (1981).

11. J.A. Erratt and A. Nasim, Mol. Gen. Genet. 202, 255-258 (1986).

12. K. Wackerbauer, T. Kossa and R. Tressl, Europ. Brew. Conv. Proc. 16th Congress, Amsterdam, Netherlands 495-505 (1977).

13. R.S. Tubb, B.A. Searle, A.R. Gooday and A.J.P. Brown, Europ. Brew. Conv. Proc. 18th Congress, Copenhagen, Denmark 487-496 (1981).

14. A.M. Sills, M.E. Sauder and G.G. Stewart, Dev. Ind. Microbiol. 24, 295-303 (1983).

15. A.M. Sills, C.J. Panchal, I. Russell and G.G. Stewart, in Proc. Alko Yeast Symposium (M. Korhola and G. Vaisanen eds.) p.209-228, Found. Biotech. Indust. Ferment. Res., Helsinki, Finland (1983).

16. A.M. Sills, I. Russell and G.G. Stewart, Europ. Brew. Conv. Proc. 19th Congress, London, England 377-384 (1983).

17. G.K. Whitney, C.R. Murray, I. Russell and G.G. Stewart, Biotech. Lett. I, 349-354 (1985).

18. I. Russell, C.M. Crumplen, R.M. Jones and G.G. Stewart, Biotech. Lett. 3 , 169-174 (1986).

19. G.G. Stewart, I. Russell and C.J. Panchal, Canadian Patent No. 1,119,593 (1986). 